\title{
Influence of a 3-months low-calorie Mediterranean diet vs. Vegetarian diet on human gut microbiota and SCFA: the CARDIVEG Study
}

\author{
Giuditta Pagliai ${ }^{1}$, Edda Russo $^{1}$, Elena Niccolai ${ }^{1}$, Monica Dinu ${ }^{1}$, Vincenzo Di Pilato ${ }^{1}$, \\ Alessandro Magrini ${ }^{1}$, Gianluca Bartolucci ${ }^{2}$, Simone Baldi ${ }^{1}$, Marta Menicatti ${ }^{2}$, Betti Giusti ${ }^{1,3}$, \\ Rossella Marcucci $^{1,3}$, Gian Maria Rossolini ${ }^{1}$, Alessandro Casini ${ }^{1,4}$, Francesco Sofi ${ }^{1,4}$ and \\ Amedeo Amedei ${ }^{1,5}$ \\ ${ }^{1}$ Department of Experimental and Clinical Medicine, University of Florence, Florence, Italy, \\ ${ }^{2}$ Department of Neurosciences, Psychology, Drug Research and Child Health Section of Pharmaceutical and \\ Nutraceutical Sciences University of Florence, Florence, Italy, \\ ${ }^{3}$ Atherothrombotic Diseases Unit, Careggi University Hospital, Florence, Italy, \\ ${ }^{4}$ Clinical Nutrition Unit, Careggi University Hospital, Florence, Italy and \\ ${ }^{5}$ Department of Biomedicine, Careggi University Hospital, Florence, Italy
}

\section{Abstract}

Introduction: There is growing interest in understanding how diet can modulate the gut microbiota (GM), including its possible association with disease states. The aim of the present study is to compare in a group of subjects in primary prevention for cardiovascular disease (CVD) the effects of Mediterranean (MD) and Vegetarian (VD) dietary patterns on the GM composition and on the shortchain fatty acids (SCFA) production.

Materials and Methods: Twenty-three clinically healthy subjects ( $16 \mathrm{~F}$; mean age: $58.6 \pm 9.8$ years) were randomly assigned to isocaloric MD or VD diets lasting 3-months each and then crossed. Anthropometric measurements, body composition, blood and fecal samples were obtained from each participant at the beginning and at the end of each intervention phase. Next Generation Sequencing (NGS) of $16 \mathrm{~S}$ rRNA were performed to analyze the GM, while the SCFA were evaluated through the Gas Chromatography-Mass Spectrometry system.

Results: Dietary interventions didn't produce significant diversity in the GM composition at higher ranks (family and above), neither between nor within MD and VD, but they did it at genus level. MD significantly changed the abundance of three genera (Enterohabdus, Lachnoclostridium and Parabacteroides), while VD significantly affected the abundance of four genera (Anaerostipes, Streptococcus, Clostridium sensu stricto and Odoribacter). Comparison of the mean variation of each SCFA between MD and VD showed an opposite and statistically significant trend for propionic acid (+ 10\% vs $-28 \%$, respectively, $\mathrm{p}=0.034)$. In addition, variations of SCFA resulted to be negatively correlated with changes of some inflammatory cytokines such as VEGF, MCP-1, IL-17, IP-10 and IL-12, only after MD. Finally, correlation analyses showed several associations between changes of genera, clinical and biochemical parameters, after both the dietary interventions.

Discussion: Our study indicates that a short-term dietary intervention with MD or VD does not induce major change in the GM, suggesting that a diet should last for longer periods to scratch the resilience of GM. In addition, the negative association between SCFA and a number of inflammatory cytokines reported only after MD, seems to support the anti-inflammatory properties of the MD. Furthermore, several associations between certain bacterial groups, clinical and biochemical parameters, let us hypothesized that the cardiovascular protection associated with the two diets could be due - at least in part - to a modulation of the GM.

\section{Conflict of Interest}

There is no conflict of interest 